\title{
Covariant GUP Deformed Hamilton-Jacobi Method
}

\author{
Benrong Mu, ${ }^{1,2}$ Peng Wang, ${ }^{2}$ and Haitang Yang ${ }^{2,3}$ \\ ${ }^{1}$ Physics Teaching and Research Section, College of Medical Technology, Chengdu University of Traditional Chinese Medicine, \\ Chengdu 611137, China \\ ${ }^{2}$ Center for Theoretical Physics, College of Physical Science and Technology, Sichuan University, Chengdu 610064, China \\ ${ }^{3}$ Kavli Institute for Theoretical Physics China (KITPC), Chinese Academy of Sciences, Beijing 100080, China \\ Correspondence should be addressed to Benrong Mu; benrongmu@cdutcm.edu.cn
}

Received 1 August 2017; Revised 20 November 2017; Accepted 26 November 2017; Published 18 December 2017

Academic Editor: Anastasios Petkou

Copyright (c) 2017 Benrong Mu et al. This is an open access article distributed under the Creative Commons Attribution License, which permits unrestricted use, distribution, and reproduction in any medium, provided the original work is properly cited. The publication of this article was funded by SCOAP $^{3}$.

\begin{abstract}
We first briefly revisit the original Hamilton-Jacobi method and show that the Hamilton-Jacobi equation for the action $I$ of tunneling of a fermionic particle from a charged black hole can be written in the same form of that for a scalar particle. On the other hand, various theories of quantum gravity suggest the existence of a minimal length scale, incorporating of which into quantum mechanics implies a modification of the uncertainty principle. In the scenario incorporating the generalized uncertainty principle (GUP) into a quantum field theory (QFT) in a covariant way, we derive the deformed model-independent KG/Dirac and Hamilton-Jacobi equations using the methods of effective field theory. For this Lorentz invariant GUP modified QFT, we find that the effect of GUP on the Hamilton-Jacobi equations is simply to "renormalize" the mass of the emitted particles, from $m$ to $m_{\text {eff }}$. Therefore, in this scenario, the Hawking temperature of a black hole does not receive any corrections from the GUP effect.
\end{abstract}

\section{Introduction}

Hawking radiation is a theoretical argument proposed by Hawking for the existence of thermal radiation emitted by black holes. The standard Hawking formula was first obtained in the frame of quantum field theory in curved spacetime [1]. Afterward, there are various methods for deriving Hawking radiation and calculating its temperature. Among them is a semiclassical method of modeling Hawking radiation as a tunneling effect. This method was first proposed by Kraus and Wilczek [2, 3]. They employed the dynamical geometry approach to calculate the imaginary part of the action for the tunneling process of s-wave emission across the horizon and related it to the Boltzmann factor for the emission at the Hawking temperature. Due to back-reaction effects included, this procedure gives a correction to the standard Hawking temperature formula, which speeds up the process of black holes' evaporation. An alternative way to calculate the imaginary part of the action is the Hamilton-Jacobi method $[4,5]$. Neglecting the self-gravitation, this method assumes that the action of an emitted particle satisfies the relativistic Hamilton-Jacobi equation. Taking the symmetries of the metric into account, one can adopt an appropriate ansatz for the form of the action. Solving the Hamilton-Jacobi equation turns out to recover the standard Hawking temperatures.

However, the original Hamilton-Jacobi method proposed in $[4,5]$ is only confined to the semiclassical approximation and carried out in the framework of the classical general relativity. Beyond the original Hamilton-Jacobi method, there could be some corrections. For simplicity, we use the case of emission of massless scalar with energy $E$ from the Schwarzschild black hole with mass $M$ to illustrate these corrections. In this paper, we set $G=c=1$, where the Planck constant $\hbar$ is of the order of square of the Planck Mass $M_{p}$. In these units, the black hole's horizon $r_{H}=2 \mathrm{M}$. The potential corrections to the original Hamilton-Jacobi method are given as follows:

(a) Back-reaction effects, which gives the correction $\sim E / M$ : In the Hamilton-Jacobi method, we assume that the metric is fixed and the field is free. Thus, back-reaction effects are disregarded. However, in Parikh and Wilczek method [2, 3], there are backreaction effects to ensure energy conservation during 
the emission of a particle via tunneling through the horizon. These corrections lead to nonthermal corrections to the black hole radiation spectrum.

(b) Higher-order WKB corrections: Using WKB approximation method, the lowest order of the equation of motion describing a particle moving in the black hole gives the Hamilton-Jacobi equation. The WKB approximation breaks down when the de Broglie wavelength of the particle, $\lambda_{p} \sim \hbar / E$, becomes comparable to the horizon of black hole, $r_{H}$. Therefore, the ratio $\lambda_{p} / r_{H} \sim \hbar / E M$ controls the amount of higherorder WKB corrections. However, several authors [68 ] argued that the tunneling method yields no higherorder corrections to the Hawking temperature.

(c) Corrections from quantum gravity effects: The original Hawking formula predicts the complete evaporation of black holes, which leads to the black hole information paradox (for reviews see $[9,10]$ ). Solving the paradox needs the breakdown of the semiclassical description at the Planck scale. A theory of quantum gravity must be used to describe the final state of black hole evaporation.

In this paper, we will investigate the quantum gravity effects on Hawking radiation in the tunneling paradigm. Although a number of quantum gravity theories are proposed, there is still no complete and consistent quantum theory of gravity. Consequently, in the absence of a full quantum description of the black hole evaporation, one uses effective models to describe the quantum gravitational behavior. On the other hand, various theories of quantum gravity, such as string theory, loop quantum gravity, and quantum geometry, predict the existence of a minimal length [11-13]. An effective model to realize this minimal length is the generalized uncertainty principle (GUP). For onedimensional quantum mechanics, the GUP,

$$
\Delta \widehat{x} \Delta \widehat{p} \geq \frac{\hbar}{2}\left(1+\beta \Delta \widehat{p}^{2}\right)
$$

can be obtained from the deformed commutation relation

$$
[\widehat{x}, \widehat{p}]=i \hbar\left(1+\beta \widehat{p}^{2}\right)
$$

where $\beta$ is a small parameter of dimension of inverse momentum squared. In fact, (1) implies an absolute minimum uncertainty of

$$
\Delta x_{\min }=\hbar \sqrt{\beta} .
$$

The operators can be given in the position representation

$$
\begin{aligned}
& \widehat{x}=x, \\
& \widehat{p}=-i \hbar \partial_{x}\left(1-\hbar^{2} \beta \partial_{x}^{2}\right)+\mathcal{O}\left(\beta^{2}\right),
\end{aligned}
$$

which is suited best for this paper. When the one-dimensional deformed commutation relation (2) is generalized to $D$ dimensions, there are several forms available in the literature
[14-17]. For example, Kempf et al. [14] modified the multipledimensional commutation relations and got

$$
\left[x_{i}, p_{j}\right]=i \hbar \delta_{i j}\left(1+\beta p^{2}\right) \text {, }
$$

where $p^{2}=\sum_{i=1}^{D} p_{i}^{2}$ and the generalization preserves the rotational symmetry. Hossenfelder et al. [15] also used modified de Broglie relation approach to get the same commutation relations. When incorporating the GUP into quantum field theory, one needs to generalize $D$-dimensional deformed commutation relations to include time. However, the existence of the minimal length could lead to Planck scale departures from Lorentz symmetry. Therefore, the corresponding $(D+1)$-dimensional deformed commutation relations are not Lorentz invariant and give rise to some version of the deformed special relativity. The field theories built on such commutation relations hence do not respect Lorentz symmetry $[15,18-20]$. Although Lorentz invariance is not required for the generalization, there are some attempts to introduce $(D+1)$-dimensional deformed Lorentz invariant generalizations of $D$-dimensional deformed commutation relations [21, 22]. Thus, the field theories based on these deformed commutation relations are Lorentz invariant. For example, the gauge theories with incorporation of the GUP in [22] are both invariant under Lorentz symmetry and gauge symmetry. In addition, the Klein-Gordon equation described by Quesne-Tkachuk Lorentz-covariant deformed algebra has been given in [23].

The standard Hawking formula predicts the complete evaporation of black holes, which leads to the black hole information paradox. There are several approaches to solve the paradox. One of them is that information might be stored in a Planck-sized remnant. This motivates Adler et al. [24] to incorporate the GUP into the derivation of Hawking temperature, which predicts the existence of black hole remnants. The black hole thermodynamics has also been discussed in the GUP framework [25-27]. Incorporating the GUP into the Parikh-Wilczek tunneling mechanism for scalar fields, the corrected Hawking temperatures in the Schwarzschild were obtained [28]. Similarly, one can also use HamiltonJacobi method to calculate effects of quantum gravity on particles' tunneling. Recently, the GUP modified HamiltonJacobi equations for fermions in curved spacetime have been introduced and the corrected Hawking temperatures have been derived [29-34].

For the Hamilton-Jacobi method, the GUP deformed Klein-Gordon (KG)/Dirac equation in $(D+1)$-dimensional curved spacetime needs to be first derived. However, different $(D+1)$-dimensional GUP deformed commutation relations could lead to different deformed KG/Dirac equation. As mentioned previously, there are two kinds of $(D+1)$ dimensional deformed commutation relations, one of which is Lorentz invariant and the other is not. The field theory built on the Lorentz invariant deformed commutation relations is also Lorentz invariant in flat spacetime. When generalized to curved spacetime using the minimal-coupling principle, the Lorentz invariant deformed Klein-Gordon (KG) equation or Dirac equation becomes covariant. On the other hand, the deformed commutation relations in [15] are not Lorentz 
invariant and hence the deformed Dirac equation built on them in $[30,31,33]$ is not covariant.

In this paper, we will study the deformed Hamilton-Jacobi method which incorporates the GUP in a covariant way. In this scenario, we will show that there are no corrections to the Hawking temperature of a black hole from the GUP effects if its original Hawking temperature is independent of the mass of emitted particles. For these purposes, this paper is organized as follows. In Section 2, we briefly review the HamiltonJacobi method and show that the fermionic Hamilton-Jacobi equation for the action $I$ can be written in the same form of the scalar one. After constructing scalar (fermionic) effective field theories respecting covariance of coordinate spacetime, we derive the deformed model-independent KG/Dirac and Hamilton-Jacobi equations in Section 3. Section 4 is devoted to our discussion and conclusion.

\section{Hamilton-Jacobi Method}

In this section, we briefly review how to calculate the imaginary part of the action making use of the Hamilton-Jacobi equation [4]. This semiclassical method models Hawking radiation as tunneling through the horizon. Using the WKB approximation the tunneling probability for the classically forbidden trajectory through the horizon is given by

$$
\Gamma \propto \exp \left(\frac{-2 \operatorname{Im} I}{\hbar}\right),
$$

where $I$ is the classical action of the trajectory. One can relate $\Gamma$ to the Boltzmann factor for the emission from the black hole to get Hawking temperature.

2.1. Scalar Field. The equation satisfied by the scalar field is

$$
\begin{aligned}
D^{\mu} D_{\mu} \phi+\frac{m^{2}}{\hbar^{2}} \phi= & \left(\nabla^{\mu}+\frac{i e}{\hbar} A^{\mu}\right)\left(\nabla_{\mu}+\frac{i e}{\hbar} A_{\mu}\right) \phi \\
& +\frac{m^{2}}{\hbar^{2}} \phi=0,
\end{aligned}
$$

where $\nabla_{\mu}$ is the covariant derivative of the black hole and $A_{\mu}$ is its electromagnetic potential. Making the ansatz for $\phi$, which is

$$
\phi=\exp \left(\frac{i I}{\hbar}\right),
$$

and substituting it into (7), one expands (7) in powers of $\hbar$ and finds the lowest order

$$
\left(\partial^{\mu} I+e A^{\mu}\right)\left(\partial_{\mu} I+e A_{\mu}\right)-m^{2}=0 .
$$

Equation (9) is just the Hamilton-Jacobi equation satisfied by a scalar particle of mass $m$ moving in the black hole with the electromagnetic potential $A_{\mu}$. The solution to (9) is the action of the scalar's classically forbidden trajectory through the horizon.

To illustrate how the Hamilton-Jacobi method works, we consider Hawking radiation in the $(1+1)$-dimensional Schwarzschild black hole with line element (with $c=G=1$ )

$$
d s^{2}=\left(1-\frac{2 M}{r}\right) d t^{2}-\left(1-\frac{2 M}{r}\right)^{-1} d r^{2} .
$$

Thus, (9) becomes

$$
\left(1-\frac{2 M}{r}\right)^{-1}\left(\partial_{t} I\right)^{2}-\left(1-\frac{2 M}{r}\right)\left(\partial_{r} I\right)^{2}-m^{2}=0,
$$

where we use $A_{\mu}=0$ for the Schwarzschild black hole. Using the method of separation of variables, we find that the solution to the above equation is

$$
I_{ \pm}=-E t \mp \int \frac{d r}{1-2 M / r} \sqrt{E^{2}-m^{2}\left(1-\frac{2 M}{r}\right)},
$$

where $E$ is a constant and $+(-)$ corresponds to the outgoing (ingoing) solutions. Choosing the contour to lie in the upper complex plane, one gets that the imaginary part of $I$ is

$$
\begin{aligned}
\operatorname{Im} I_{ \pm} & =\mp \int_{2 M-\epsilon}^{2 M+\epsilon} \frac{d r}{1-2 M / r} \sqrt{E^{2}-m^{2}\left(1-\frac{2 M}{r}\right)} \\
& = \pm 2 \pi E M .
\end{aligned}
$$

Thus, the tunneling rate is

$$
\Gamma=\frac{P_{(\text {emission })}}{P_{(\text {absorption })}}=\frac{\exp \left(-2 \operatorname{Im} I_{+}\right)}{\exp \left(-2 \operatorname{Im} I_{-}\right)}=\exp (-8 \pi M E) .
$$

Comparing the above equation with the Boltzmann factor at the Hawking temperature near the event horizon gives

$$
T=\frac{1}{8 \pi M} .
$$

2.2. Fermion Field. In curved spacetime, the Dirac equation for a spin-1/2 fermion with an electromagnetic field $A_{\mu}$ takes on the form

$$
i \gamma_{\mu}\left(\partial^{\mu}+\Omega^{\mu}+\frac{i e}{\hbar} A_{\mu}\right) \psi-\frac{m}{\hbar} \psi=0,
$$

where $\Omega_{\mu} \equiv(i / 2) \omega_{\mu}{ }^{a b} \Sigma_{a b}, \Sigma_{a b}$ is the Lorentz spinor generator, $\omega_{\mu}{ }^{a b}$ is the spin connection, and $\left\{\gamma_{\mu}, \gamma_{\nu}\right\}=2 g_{\mu \nu}$. The Greek indices are raised and lowered by the curved metric $g_{\mu \nu}$, while the Latin indices are governed by the flat metric $\eta_{a b}$. To obtain the Hamilton-Jacobi equation for a fermion, the ansatz for $\psi$ is assumed as

$$
\psi=\exp \left(\frac{i I}{\hbar}\right) v
$$

where $v$ is a slowly varying spinor amplitude. Substituting (17) into (16), we find to the lowest order of $\hbar$

$$
\gamma_{\mu}\left(\partial^{\mu} I+e A^{\mu}\right) v=-m v,
$$

which is the Hamilton-Jacobi equation satisfied by a fermion particle of mass $m$ moving in the black hole with the electromagnetic potential $A_{\mu}$. Solving (18) gives us the classical action $I$. Multiplying both sides of (18) from the left by $\gamma_{\nu}\left(\partial^{v} I+e A^{v}\right)$ and then using (18) to simplify the RHS, one gets

$$
\gamma_{\nu}\left(\partial^{v} I+e A^{v}\right) \gamma_{\mu}\left(\partial^{\mu} I+e A^{\mu}\right) v=m^{2} v
$$


Manipulating the LHS of the above equation by using $\left\{\gamma_{\mu}, \gamma_{\nu}\right\}=2 g_{\mu \nu}$, we have

$$
\left[\left(\partial^{\mu} I+e A^{\mu}\right)\left(\partial_{\mu} I+e A_{\mu}\right)-m^{2}\right] v=0
$$

Since $v$ is nonzero, the Hamilton-Jacobi equation satisfied by the classical action $I$ for a fermion finally becomes

$$
\left(\partial^{\mu} I+e A^{\mu}\right)\left(\partial_{\mu} I+e A_{\mu}\right)-m^{2}=0
$$

which is the same as the Hamilton-Jacobi equation for a scalar with the same mass $m$, namely, (9).

\section{Hamilton-Jacobi Equation Incorporating GUP}

To incorporate the GUP into the Hamilton-Jacobi method, we need to derive the KG/Dirac equations in the framework of the GUP. As mentioned in the Introduction, there are a lot of attempts to introduce the GUP into quantum field theory. If the field theory is built on the Lorentz invariant deformed commutation relations, the deformed KG/Dirac equations are covariant in curved spacetime. In this section, we will calculate the Hawking radiation in the framework of the covariant deformed KG/Dirac equations by making use of the Hamilton-Jacobi method.

3.1. Model-Independent Covariant Deformed KG/Dirac Equations. The forms of the covariant deformed KG/Dirac equations depend on the deformed commutation relations which we use. To be as general as possible, in this paper we will not resort to any specific model. Instead, we will derive the model-independent covariant deformed KG/Dirac equations by considering effective field theories respecting covariance. For the charged black hole background, the $U(1)$ gauge invariance of the effective field theory is assumed as well. We also assume introducing the GUP to the original field theory does not make a stable particle unstable. Now that the original field theory is free, there are no self-interacting effective operators in the effective field theories. Thus, the effective Lagrangian involving the scalar field $\phi$ (fermion field $\psi)$ in the background of a $(D+1)$-dimensional black hole with the electromagnetic potential $A_{\mu}$ is given by

$$
\mathscr{L}_{\mathrm{eff}}^{s(f)}=\sum_{n, j} \frac{C_{n, j}^{s(f)}}{\Lambda^{n-(D+1)}} \mathcal{O}_{n, j}^{s(f)},
$$

where $s(f)$ denotes the scalar (fermion), the $n \geq D+1$ denotes the operator dimension, $j$ runs over all independent operators of a given dimension, and $\Lambda$ is the energy scale related to the minimal length $l_{\min }, \Lambda \sim \hbar / l_{\min }$. The lowest dimensional operator with $n=D+1$ is the original free field Lagrangian in curved spacetime with the electromagnetic potential $A_{\mu}$ :

$$
\begin{aligned}
& \mathcal{O}_{D+1}^{s}=-\phi^{+}\left(D^{\mu} D_{\mu}+\frac{m^{2}}{\hbar^{2}}\right) \phi, \\
& \mathcal{O}_{D+1}^{f}=\bar{\psi}\left[i D_{\mu}^{f} \gamma^{\mu}-\frac{m}{\hbar}\right] \psi,
\end{aligned}
$$

where $D_{\mu} \equiv \nabla^{\mu}+(i e / \hbar) A^{\mu}, D_{\mu}^{f} \equiv \partial_{\mu}+\Omega_{\mu}+(i e / \hbar) A_{\mu}$, and $m$ is the mass of the particle. For the fermion field $\psi$, the basis of independent effective operators with $n=D+2$ is given by

$$
\begin{aligned}
& \mathcal{O}_{D+2,1}^{f}=\hbar \bar{\psi}\left(\gamma^{\nu} D_{\nu}^{f}\right)\left(\gamma^{\mu} D_{\mu}^{f}\right) \psi, \\
& \mathcal{O}_{D+2,2}^{f}=\hbar \bar{\psi} D^{f, \mu} D_{\mu}^{f} \psi, \\
& \mathcal{O}_{D+2,3}^{f}=i m \bar{\psi}\left(\gamma^{\mu} D_{\mu}^{f}\right) \psi, \\
& \mathcal{O}_{D+2,4}^{f}=\frac{m^{2}}{\hbar} \bar{\psi} \psi .
\end{aligned}
$$

For the scalar field $\phi$, the operator with $n=D+2$ which are gauge-invariant and covariant is

$$
\mathcal{O}_{D+2}^{s}=m \phi^{+}\left(D^{\mu} D_{\mu}\right) \phi .
$$

If we truncate the scalar effective theory at $\mathcal{O}(1 / \Lambda)$, by redefining the scalar field $\phi$, it is easy to see that the truncated effective theory is equivalent to the original field theory with redefined mass. So we need effective operators at $\mathcal{O}\left(1 / \Lambda^{2}\right)$ to produce nontrivial results. The basis of independent effective operators with $n=D+3$ is

$$
\begin{aligned}
& \mathcal{O}_{D+3,1}^{s}=\hbar^{2} \phi^{+}\left(D^{\mu} D_{\mu} D^{\nu} D_{\nu}\right) \phi, \\
& \mathcal{O}_{D+3,2}^{s}=\hbar^{2} \phi^{+}\left(D^{\mu} D^{\nu} D_{\nu} D_{\mu}\right) \phi, \\
& \mathcal{O}_{D+3,3}^{s}=\hbar^{2} \phi^{+}\left(D^{\mu} D^{\nu} D_{\mu} D_{\nu}\right) \phi, \\
& \mathcal{O}_{D+3,4}^{s}=m^{2} \phi^{+}\left(D^{\mu} D_{\mu}\right) \phi, \\
& \mathcal{O}_{D+3,5}^{s}=\frac{m^{4}}{\hbar^{2}} \phi^{+} \phi .
\end{aligned}
$$

Define the action $S_{\text {eff }}^{s(f)}=\int d^{D+1} x \sqrt{|g|} \mathscr{L}_{\text {eff }}^{s(f)}$. Varying $S_{\text {eff }}^{s(f)}$ with respect to $\phi^{+}(\bar{\psi})$ gives the deformed KG (Dirac) equation of $\phi(\psi)$. Thus, the deformed KG equation to $\mathcal{O}\left(1 / \Lambda^{2}\right)$ is

$$
\begin{aligned}
& -D^{\mu} D_{\mu} \phi-\frac{m^{2}}{\hbar^{2}} \phi+C_{D+2}^{s} \frac{m}{\Lambda} D^{\mu} D_{\mu} \phi \\
& +\frac{C_{D+3,1}^{s} \hbar^{2}}{\Lambda^{2}} D^{\mu} D_{\mu} D^{\nu} D_{\nu} \phi \\
& +\frac{C_{D+3,2}^{s} \hbar^{2}}{\Lambda^{2}} D^{\mu} D^{\nu} D_{\nu} D_{\mu} \phi \\
& +\frac{C_{D+3,3}^{s} \hbar^{2}}{\Lambda^{2}} D^{\mu} D^{\nu} D_{\mu} D_{\nu} \phi+\frac{C_{D+3,4}^{s} m^{2}}{\Lambda^{2}} D^{\mu} D_{\mu} \phi \\
& +\frac{C_{D+3,5}^{s}}{\Lambda^{2}} \frac{m^{4}}{\hbar^{2}} \phi=0,
\end{aligned}
$$

and the deformed Dirac equation to $\mathcal{O}(1 / \Lambda)$ is

$$
i \gamma^{\mu} D_{\mu}^{f} \psi-\frac{m}{\hbar} \psi+\frac{C_{D+2,1}^{f} \hbar}{\Lambda}\left(\gamma^{\nu} D_{\nu}^{f}\right)\left(\gamma^{\mu} D_{\mu}^{f}\right) \psi
$$




$$
\begin{aligned}
& +\frac{C_{D+2,2}^{f} \hbar}{\Lambda} D^{f, \mu} D_{\mu}^{f} \psi+\frac{i C_{D+2,3}^{f} m}{\Lambda}\left(\gamma^{\mu} D_{\mu}^{f}\right) \psi \\
& +\frac{C_{D+2,4}^{f}}{\Lambda} \frac{m^{2}}{\hbar} \psi=0 .
\end{aligned}
$$

3.2. Deformed Scalar Hamilton-Jacobi Equation. To find the classical action $I$ by using WKB approximation to solve (27), we make the ansatz for $\phi$ as before

$$
\phi=\exp \left(\frac{i I}{\hbar}\right) .
$$

Substituting it into (27), one expands (27) in powers of $\hbar$ and finds the lowest order

$$
\begin{aligned}
& A\left(\partial^{\mu} I+e A^{\mu}\right)\left(\partial_{\mu} I+e A_{\mu}\right)-B m^{2} \\
& \quad+\frac{C}{\Lambda^{2}}\left[\left(\partial^{\mu} I+e A^{\mu}\right)\left(\partial_{\mu} I+e A_{\mu}\right)\right]^{2}=0,
\end{aligned}
$$

where $A=1-C_{D+2}^{s} m / \Lambda-C_{D+3,4}^{s} m^{2} / \Lambda^{2}, B=1+$ $C_{D+3,5}^{s} m^{2} / \Lambda^{2}$, and $C \equiv C_{D+3,1}^{s}+C_{D+3,2}^{s}+C_{D+3,3}^{s}$. Solving (30) gives

$$
\left(\partial^{\mu} I+e A^{\mu}\right)\left(\partial_{\mu} I+e A_{\mu}\right)=m_{\mathrm{eff}, \pm}^{2},
$$

where

$$
m_{\mathrm{eff}, \pm}^{2} \equiv \frac{-A \pm \sqrt{A^{2}+4 B C m^{2} \Lambda^{-2}}}{2 C} \Lambda^{2} .
$$

When $m / \Lambda \rightarrow 0$, we find $m_{\text {eff, },+}^{2} \sim m^{2}$ and $m_{\text {eff,-- }}^{2} \sim-(2 / C) \Lambda^{2}$. As $\Lambda \gg m$, (31) gives $\partial^{\mu} I \sim \Lambda$ for $m_{\text {eff,- }}^{2}$ and hence the action $I$ highly oscillates in spacetime. One may argue such action is not physical and hence could be discarded by using the low-momentum consistency condition proposed in [35]. Alternatively, one wants to recover the results without the GUP when $\Lambda \rightarrow \infty$. However, as $\Lambda \rightarrow \infty$, the solution to (31) with $m_{\text {eff,- }}^{2}$ blows up while (31) with $m_{\text {eff, }}^{2}$ becomes (9) since $m_{\mathrm{eff},+}^{2} \rightarrow m^{2}$. Therefore, we pick $m_{\mathrm{eff},+}^{2}$ in (31). Comparing (31) with (9), we find that all the GUP contributions to the deformed scalar Hamilton-Jacobi equation are included in one effective parameter, $m_{\mathrm{eff}}^{2}$.

3.3. Deformed Fermionic Hamilton-Jacobi Equation. To obtain the Hamilton-Jacobi equation for the classical action, the ansatz for $\psi$ takes the form of

$$
\psi=\exp \left(\frac{i I}{\hbar}\right) v
$$

where $v$ is a vector function of the spacetime. Substituting (33) into (28), we find the lowest order of $\hbar$

$$
\begin{aligned}
- & A \gamma_{\mu}\left(\partial^{\mu} I+e A^{\mu}\right) v \\
& =\left[B m+\frac{C}{\Lambda}\left(\partial^{\mu} I+e A^{\mu}\right)\left(\partial_{\mu} I+e A_{\mu}\right)\right] v,
\end{aligned}
$$

where $A=1+C_{D+2,3}^{f} m / \Lambda, B=1+C_{D+2,4}^{f} m / \Lambda$, and $C \equiv$ $C_{D+2,1}^{f}+C_{D+2,2}^{f}$. Multiplying both sides of (34) from the left by $-A \gamma_{\nu}\left(\partial^{\nu} I+e A^{\nu}\right)$ and using (34) on RHS, we obtain

$$
\begin{aligned}
A^{2} & \left(\partial^{\mu} I+e A^{\mu}\right)\left(\partial_{\mu} I+e A_{\mu}\right) \\
\quad & =\left[B m+\frac{C}{\Lambda}\left(\partial^{\mu} I+e A^{\mu}\right)\left(\partial_{\mu} I+e A_{\mu}\right)\right]^{2} .
\end{aligned}
$$

Solving (35), we also find

$$
\left(\partial^{\mu} I+e A^{\mu}\right)\left(\partial_{\mu} I+e A_{\mu}\right)=m_{\mathrm{eff}}^{2},
$$

where $m_{\text {eff }}^{2}$ is a function of $m, \Lambda, A, B$, and $C$ and is chosen as $m_{\text {eff }}^{2} \rightarrow m^{2}$ as $\Lambda \rightarrow \infty$. Similarly, all the GUP contributions to the deformed fermionic Hamilton-Jacobi equation are included in one effective parameter, $m_{\text {eff }}^{2}$.

3.4. Deformed Hamilton-Jacobi Equation to All Orders. We have shown that the deformed scalar (fermionic) HamiltonJacobi equation can be written as the form of (36) up to the order of $\left(1 / \Lambda^{2}\right)(1 / \Lambda)$. Here, we will show that the deformed scalar (fermionic) Hamilton-Jacobi equation can also be written as the form of (36) to all orders of the effective theories.

For a scalar field, the effective operator $\mathscr{O}_{n, j}^{s}$ must contain even number of $D_{\mu}$ to be covariant. Since $\mathscr{O}_{n, j}^{s}$ contains two $\phi$, we find

$$
\mathcal{O}_{n, j}^{s}=\hbar^{2 q-2} m^{p} \phi^{+} \mathscr{C}\left(D_{\mu_{1}} \cdots D_{\mu_{2 q}}\right) \phi,
$$

where integers $p, q \geqslant 0,2 q+p=n-D+1, j=\{\mathscr{C}, p\}$ and $\mathscr{C}$ denotes any possible way of contracting $\mu_{1} \cdots \mu_{2 q}$ in pair to make $\mathcal{O}_{n, j}^{s}$ covariant. Therefore, the deformed KG equation to all orders of the effective theory is

$$
\begin{aligned}
-D^{\mu} D_{\mu} \phi-\frac{m^{2}}{\hbar^{2}} \phi & \\
& +\sum_{j, n>D+1} C_{n, j}^{s} \frac{\hbar^{2 q-2} m^{p}}{\Lambda^{n-(D+1)}} \mathscr{C}\left(D_{\mu_{1}} \cdots D_{\mu_{2 q}}\right) \phi=0 .
\end{aligned}
$$

Making the ansatz $\phi=\exp (i I / \hbar)$ and substituting it into (36) gives the deformed Hamilton-Jacobi equation for $I$ :

$$
G(X) \equiv X-m^{2}+f(X)=0,
$$

where $X \equiv\left(\partial^{\mu} I+e A^{\mu}\right)\left(\partial_{\mu} I+e A_{\mu}\right)$ and

$$
f(x) \equiv \sum_{j, n>D+1} \frac{(-1)^{q} C_{n, j}^{s} m^{p} x^{q}}{\Lambda^{n-(D+1)}} .
$$

When $m / \Lambda \ll 1$ as assumed, for (39), there exists one and only one root $m_{\text {eff }}^{2}$ in $\left(0,2 m^{2}\right)$. In fact, since $m / \Lambda \ll 1, f(0)$ and $f\left(2 m^{2}\right) \sim m^{2}(m / \Lambda) \ll m^{2}$. Thus, one finds $G(0)<0$ and $G\left(2 m^{2}\right)>0$. So there exists one root of (39) in $\left(0,2 m^{2}\right)$. On the other hand, using $f^{\prime}(x) \sim m / \Lambda \ll 1$ for $x \in\left(0,2 m^{2}\right)$, one 
finds $G^{\prime}(x)>0$ for $x \in\left(0,2 m^{2}\right)$. This completes the proof of existence of one and only one root of (39) in $\left(0,2 \mathrm{~m}^{2}\right)$. There might be other roots which are not in $\left(0,2 \mathrm{~m}^{2}\right)$. However, they are not physical and discarded since they do not approach $m^{2}$ as $\Lambda \rightarrow \infty$. By solving (39), we find that the classical action $I$ satisfies

$$
\left(\partial^{\mu} I+e A^{\mu}\right)\left(\partial_{\mu} I+e A_{\mu}\right)=m_{\mathrm{eff}}^{2},
$$

where $m_{\mathrm{eff}}^{2}$ is uniquely determined by $\Lambda, m$, and $C_{n, j}^{s}$.

For a fermion field, the effective operator $\mathcal{O}_{n, j}^{f}$ can be written as

$$
\mathcal{O}_{n, j}^{f}=i^{q} \hbar^{q-1} m^{p} \bar{\psi} \mathscr{C}\left(D_{\mu_{1}}^{f} \cdots D_{\mu_{q}}^{f} \gamma_{\nu_{1}} \cdots \gamma_{\nu_{2 r-q}}\right) \psi,
$$

where integers $q, p, r \geqslant 0, p+q=n-D, q \geqslant r \geqslant q / 2, j=$ $\{\mathscr{C}, q, r\}$ and $\mathscr{C}$ denotes any possible way of contracting $\mu_{1} \cdots \mu_{q}$ and $\nu_{1} \cdots v_{2 r-q}$ in pair. Therefore, the deformed Dirac equation to all orders of the effective theory is

$$
\begin{aligned}
& i \gamma^{\mu} D_{\mu}^{f} \psi-\frac{m}{\hbar} \psi \\
& +\sum_{j, n>D+1} C_{n, j}^{f} \frac{i^{q} \hbar^{q-1} m^{p}}{\Lambda^{n-(D+1)}} \mathscr{C}\left(D_{\mu_{1}}^{f} \cdots D_{\mu_{q}}^{f} \gamma_{\nu_{1}} \cdots \gamma_{\nu_{2 r-q}}\right) \psi \\
& \quad=0 .
\end{aligned}
$$

Substituting the ansatz $\psi=\exp (i I / \hbar) v$ into (43), we find to the lowest order of $\hbar$ :

$$
[1-g(X)] \gamma_{\mu}\left(\partial^{\mu} I+e A^{\mu}\right) v=[f(X)-m] v,
$$

where $X \equiv\left(\partial^{\mu} I+e A^{\mu}\right)\left(\partial_{\mu} I+e A_{\mu}\right)$ and

$$
\begin{aligned}
& f(x) \equiv \sum_{j, n>D+1, q \text { is even }} \frac{C_{n, j}^{f} m^{p} x^{q / 2}}{\Lambda^{n-(D+1)}}, \\
& g(x) \equiv \sum_{j, n>D+1, q \text { is odd }} \frac{-C_{n, j}^{f} m^{p} x^{(q-1) / 2}}{\Lambda^{n-(D+1)}} .
\end{aligned}
$$

Using the same manipulation as before, we find that (44) reduces to

$$
H(X) \equiv[1-g(X)]^{2} X-[f(X)-m]^{2}=0 .
$$

For $m / \Lambda \ll 1$, one can show that $f(0), f\left(2 m^{2}\right) \ll m$ and $g(0)$, $g\left(2 m^{2}\right) \ll 1$. Thus, $H(0)<0$ and $H\left(2 m^{2}\right)>0$. Moreover, $H^{\prime}(x)>0$ for $x \in\left(0,2 m^{2}\right)$ since $f^{\prime}(x) \ll 1$ and $x g^{\prime}(x) \ll 1$ for $x \in\left(0,2 m^{2}\right)$. Therefore, there exists one and only one root $m_{\mathrm{eff}}^{2}$ of $(46)$ in $\left(0,2 m^{2}\right)$. (46) leads to

$$
\left(\partial^{\mu} I+e A^{\mu}\right)\left(\partial_{\mu} I+e A_{\mu}\right)=m_{\mathrm{eff}}^{2},
$$

where $m_{\mathrm{eff}}^{2}$ is uniquely determined by $\Lambda, m$, and $C_{n, j}^{f}$.

\section{Discussion and Conclusion}

The Hamilton-Jacobi method without and with the incorporation of the GUP has been studied in this paper. Specifically, we have calculated the scalar and fermionic Hamilton-Jacobi equations for the classical action $I$ in the background of a $(D+1)$-dimensional black hole with the metric $g_{\mu \nu}$ and the electromagnetic potential $A_{\mu}$. First, in Section 2, we revisited the derivation of the usual Hamilton-Jacobi equations for the action $I$ of tunneling of scalar and fermionic particles from the black hole. In the framework of effective field theories expanded in powers of $1 / \Lambda$ constructed in Section 3, the deformed model-independent KG/Dirac equations respecting covariance and gauge invariance of $A_{\mu}$ have then been derived. Finally, substituting the WKB ansatz for the scalar and fermionic wavefunctions into the deformed KG/Dirac equations, we expanded them in powers of $\hbar$, kept only the lowest order, and hence gave the deformed Hamilton-Jacobi equations. The deformed scalar (fermionic) Hamilton-Jacobi equations to $\mathcal{O}\left(1 / \Lambda^{2}\right)(\mathcal{O}(1 / \Lambda))$ are derived in Section 3 while the deformed Hamilton-Jacobi equations to all orders of the effective field theories are also given there.

Our results are summarized as follows:

(a) In the case of no GUP, we have shown in Section 2 that the fermionic Hamilton-Jacobi equation for the action $I$ can be written in the same form of the scalar one. Both can be written as

$$
\left(\partial^{\mu} I+e A^{\mu}\right)\left(\partial_{\mu} I+e A_{\mu}\right)=m^{2},
$$

where $A^{\mu}$ is the black hole's electromagnetic potential and $m$ is the mass of the particle.

(b) In the case of incorporating the GUP into quantum field theory in a covariant way, we have shown in Section 3 that both scalar and fermionic deformed Hamilton-Jacobi equations can be reduced to

$$
\left(\partial^{\mu} I+e A^{\mu}\right)\left(\partial_{\mu} I+e A_{\mu}\right)=m_{\mathrm{eff}}^{2},
$$

where all the GUP contributions are included in only one parameter $m_{\mathrm{eff}}^{2}$.

As a bonus of result (a), it provides a shortcut to calculate the action $I$ of tunneling of a fermionic particle from the black hole. Instead of solving the complicated matrix equation (18), we can solve (48) for I. For example, such shortcut was discussed in the case of fermion tunneling from the Bardeen-Vaidya black hole in [36]. Since both scalar and fermion actions satisfy the same equation (48), the HamiltonJacobi method relating the imaginary part of the actions to Hawking temperature indicates that Hawking temperatures for scalar and fermion particles are the same. In fact, using the Hamilton-Jacobi method, Hawking temperatures were calculated for a scalar and a fermion, in the context of charged BTZ black holes [37], black strings [38, 39], and so forth, and same results were found. As shown above, the coincidence is guaranteed by (48).

For the standard Hawing radiation, all particles very close to the horizon are effectively massless on account 
of infinite blueshift. Thus, the conformal invariance of the horizon makes Hawing temperatures of all particles the same. The mass, angular momentum, and identity of the particles are only relevant when they escape the potential barrier. This is what our result (a) gives. However, if quantum gravity effects are considered, behaviors of particles near the horizon could be different. For example, if we send a wave packet which is governed by a subluminal dispersion relation, backwards in time toward the horizon, it reaches a minimum distance of approach, then reverses direction, and propagates back away from the horizon, instead of getting unlimited blueshift toward the horizon [40, 41]. Thus, quantum gravity effects might make fermions and scalars experience different (effective) Hawking temperatures. However, our result shows that in the covariant GUP models, the Hawing temperatures of scalars and fermions are the same, independently of their angular momentums and identities.

When incorporating the GUP into quantum field theory, as mentioned in the Introduction, there are two kinds of realizations, one of which respects covariance and the other does not. For the noncovariant realization, the deformed Hamilton-Jacobi method incorporated with the GUP was studied in [29-34]. There, it has been shown that the corrections to the standard Hawking temperature depend on the masses and angular momentums of the emitted particles in a nontrivial way. In some cases, such corrections could lead to the remnant being left in the evaporation [29-31].

In this paper, we investigated the deformed HamiltonJacobi method for a GUP modified QFT, which incorporates the GUP in a covariant way. For this Lorentz invariant GUP modified QFT, we found that the effect of GUP on the Hamilton-Jacobi equations was simply to "renormalize" the mass of the emitted particles, from $m$ to $m_{\text {eff. Note that the }}$ original Hawking temperature is independent of the mass of emitted particles. As a consequence, there is no correction from the GUP to the Hawking temperature for a Lorentz invariant GUP modified QFT. Therefore, one may argue that covariance of incorporating the GUP into quantum field theory has to be broken in order to have the remnant left in the evaporation.

\section{Conflicts of Interest}

The authors declare that there are no conflicts of interest regarding the publication of this paper.

\section{Acknowledgments}

The authors are grateful to Houwen Wu and Zheng Sun for useful discussions. This work is supported in part by NSFC (Grants nos. 11005016, 11175039, 11375121, and 11747171) and Natural Science Foundation of Chengdu University of TCM (Grants nos. ZRYY1729 and ZRQN1656).

\section{References}

[1] S. W. Hawking, "Particle creation by black holes," Communications in Mathematical Physics, vol. 43, no. 3, pp. 199-220, 1975.
[2] P. Kraus and F. Wilczek, "Effect of self-interaction on charged black hole radiance," Nuclear Physics B, vol. 437, no. 1, pp. 231242, 1995.

[3] P. Kraus and F. Wilczek, "Self-interaction correction to black hole radiance," Nuclear Physics B, vol. 433, no. 2, pp. 403-420, 1995.

[4] M. Angheben, M. Nadalini, L. Vanzo, and S. Zerbini, "Hawking radiation as tunneling for extremal and rotating black holes," Journal of High Energy Physics, vol. 2005, no. 5, article 014, 2005.

[5] R. Kerner and R. B. Mann, "Tunnelling, temperature, and Taub-NUT black holes," Physical Review D: Particles, Fields, Gravitation and Cosmology, vol. 73, no. 10, Article ID 104010, 2006.

[6] A. Yale, "Exact Hawking radiation of scalars, fermions, and bosons using the tunneling method without back-reaction," Physics Letters B, vol. 697, no. 4, pp. 398-403, 2011.

[7] B. Chatterjee and P. Mitra, "Hawking temperature and higher order calculations," Physics Letters B, vol. 675, no. 2, pp. 240 242, 2009.

[8] M. Wang, C. Ding, S. Chen, and J. Jing, "Is Hawking temperature modified by the quantum tunneling beyond semiclassical approximation," General Relativity and Gravitation, vol. 42, no. 2, pp. 347-357, 2010.

[9] J. Preskill, Do Black Holes Destroy Information?, arXiv:hepth/9209058, 1992.

[10] C. T. Hill, The Information Problem in Black Hole Evaporation: Old and Recent Results, arXiv:hep-th/0501132, 2005.

[11] P. K. Townsend, "Small-scale structure of spacetime as the origin of the gravitational constant," Physical Review D: Particles, Fields, Gravitation and Cosmology, vol. 15, no. 10, pp. 2795-2801, 1977.

[12] D. Amati, M. Ciafaloni, and G. Veneziano, "Can spacetime be probed below the string size?" Physics Letters B, vol. 216, no. 1-2, pp. 41-47, 1989.

[13] K. Konishi, G. Paffuti, and P. Provero, "Minimum physical length and the generalized uncertainty principle in string theory," Physics Letters B, vol. 234, no. 3, pp. 276-284, 1990.

[14] A. Kempf, G. Mangano, and R. B. Mann, "Hilbert space representation of the minimal length uncertainty relation," Physical Review D: Particles, Fields, Gravitation and Cosmology, vol. 52, no. 2, pp. 1108-1118, 1995.

[15] S. Hossenfelder, M. Bleicher, S. Hofmann, J. Ruppert, S. Scherer, and H. Stöcker, "Signatures in the Planck regime," Physics Letters $B$, vol. 575, no. 1-2, pp. 85-99, 2003.

[16] F. Brau, "Minimal length uncertainty relation and the hydrogen atom," Journal of Physics A: Mathematical and General, vol. 32, no. 44, pp. 7691-7696, 1999.

[17] A. F. Ali, S. Das, and E. C. Vagenas, "Discreteness of space from the generalized uncertainty principle," Physics Letters B, vol. 678, no. 5, pp. 497-499, 2009.

[18] A. Camacho, "Generalized uncertainty principle and quantum electrodynamics," General Relativity and Gravitation, vol. 35, no. 7, pp. 1153-1160, 2003.

[19] M. Maziashvili, "Implications of minimum-length deformed quantum mechanics for QFT/QG," Fortschritte der Physik/ Progress of Physics, vol. 61, no. 6, pp. 685-700, 2013.

[20] M. S. Berger and M. Maziashvili, "Free particle wave function in light of the minimum-length deformed quantum mechanics and some of its phenomenological implications," Physical Review D: Particles, Fields, Gravitation and Cosmology, vol. 84, no. 4, Article ID 044043, 2011. 
[21] C. Quesne and V. M. Tkachuk, "Lorentz-covariant deformed algebra with minimal length and application to the $(1+1)$ dimensional Dirac oscillator," Journal of Physics A: Mathematical and General, vol. 39, no. 34, article no. 021, pp. 10909-10922, 2006.

[22] M. Kober, "Gauge theories under incorporation of a generalized uncertainty principle," Physical Review D: Particles, Fields, Gravitation and Cosmology, vol. 82, no. 8, Article ID 085017, 2010.

[23] S. K. Moayedi, M. R. Setare, and H. Moayeri, "Quantum Gravitational Corrections to the Real Klein-Gordon Field in the Presence of a Minimal Length," International Journal of Theoretical Physics, vol. 49, no. 9, pp. 2080-2088, 2010.

[24] R. J. Adler, P. Chen, and D. I. Santiago, "The generalized uncertainty principle and black hole remnants," General Relativity and Gravitation, vol. 33, no. 12, pp. 2101-2108 (2002), 2001.

[25] L. Xiang and X. Q. Wen, "Black hole thermodynamics with generalized uncertainty principle," Journal of High Energy Physics, vol. 2009, no. 10, article 46, 2009.

[26] A. Bina, S. Jalalzadeh, and A. Moslehi, "Quantum black hole in the generalized uncertainty principle framework," Physical Review D: Particles, Fields, Gravitation and Cosmology, vol. 81, no. 2, Article ID 023528, 2010.

[27] Y.-W. Kim and Y.-J. Park, "Entropy of the Schwarzschild black hole to all orders in the Planck length," Physics Letters B, vol. 655, no. 3-4, pp. 172-177, 2007.

[28] K. Nozari and S. Saghafi, "Natural cutoffs and quantum tunneling from black hole horizon," Journal of High Energy Physics, vol. 2012, no. 11, article 005, 2012.

[29] D. Chen, H. Wu, and H. Yang, "Fermion's tunnelling with effects of quantum gravity," Advances in High Energy Physics, vol. 2013, Article ID 432412, 6 pages, 2013.

[30] D. Y. Chen, H. W. Wu, and H. Yang, "Observing remnants by fermions' tunneling," Journal of Cosmology and Astroparticle Physics, no. 3, 036, front matter+12 pages, 2014.

[31] D. Y. Chen, Q. Q. Jiang, P. Wang, and H. Yang, "Remnants, fermions' tunnelling and effects of quantum gravity," Journal of High Energy Physics, vol. 2013, no. 11, pp. 1-13, 2013.

[32] D. Chen, “Dirac particles' tunneling from five-dimensional rotating black strings influenced by the generalized uncertainty principle," The European Physical Journal C, vol. 74, no. 1, article no. 2687, pp. 1-5, 2014.

[33] Z.-Y. Liu and J.-R. Ren, "Fermions tunnelling with quantum gravity correction," Communications in Theoretical Physics, vol. 62, no. 6, pp. 819-823, 2014.

[34] D. Chen and Z. Li, "Remarks on remnants by fermions' tunnelling from black strings," Advances in High Energy Physics, vol. 2014, Article ID 620157, 9 pages, 2014.

[35] C. L. Ching and R. Parwani, Scattering and Bound States of a Deformed Quantum Mechanics, arXiv:1207.1519, 2012.

[36] R. Di Criscienzo and L. Vanzo, "Fermion tunneling from dynamical horizons," EPL (Europhysics Letters), vol. 82, no. 6, Article ID 60001, 2008.

[37] A. Ejaz, H. Gohar, H. Lin, K. Saifullah, and S.-T. Yau, "Quantum tunneling from three-dimensional black holes," Physics Letters $B$, vol. 726, no. 4-5, pp. 827-833, 2013.

[38] J. Ahmed and K. Saifullah, "Hawking temperature of rotating charged black strings from tunneling," Journal of Cosmology and Astroparticle Physics, vol. 2011, no. 11, article no. 023, 2011.

[39] H. Gohar and K. Saifullah, "Emission of scalar particles from cylindrical black holes," Astrophysics and Space Science, vol. 343, no. 1, pp. 181-185, 2013.
[40] W. G. Unruh, "Sonic analogue of black holes and the effects of high frequencies on black hole evaporation," Physical Review D: Particles, Fields, Gravitation and Cosmology, vol. 51, no. 6, pp. 2827-2838, 1995.

[41] S. Corley and T. Jacobson, "Hawking spectrum and high frequency dispersion," Physical Review D: Particles, Fields, Gravitation and Cosmology, vol. 54, no. 2, pp. 1568-1586, 1996. 

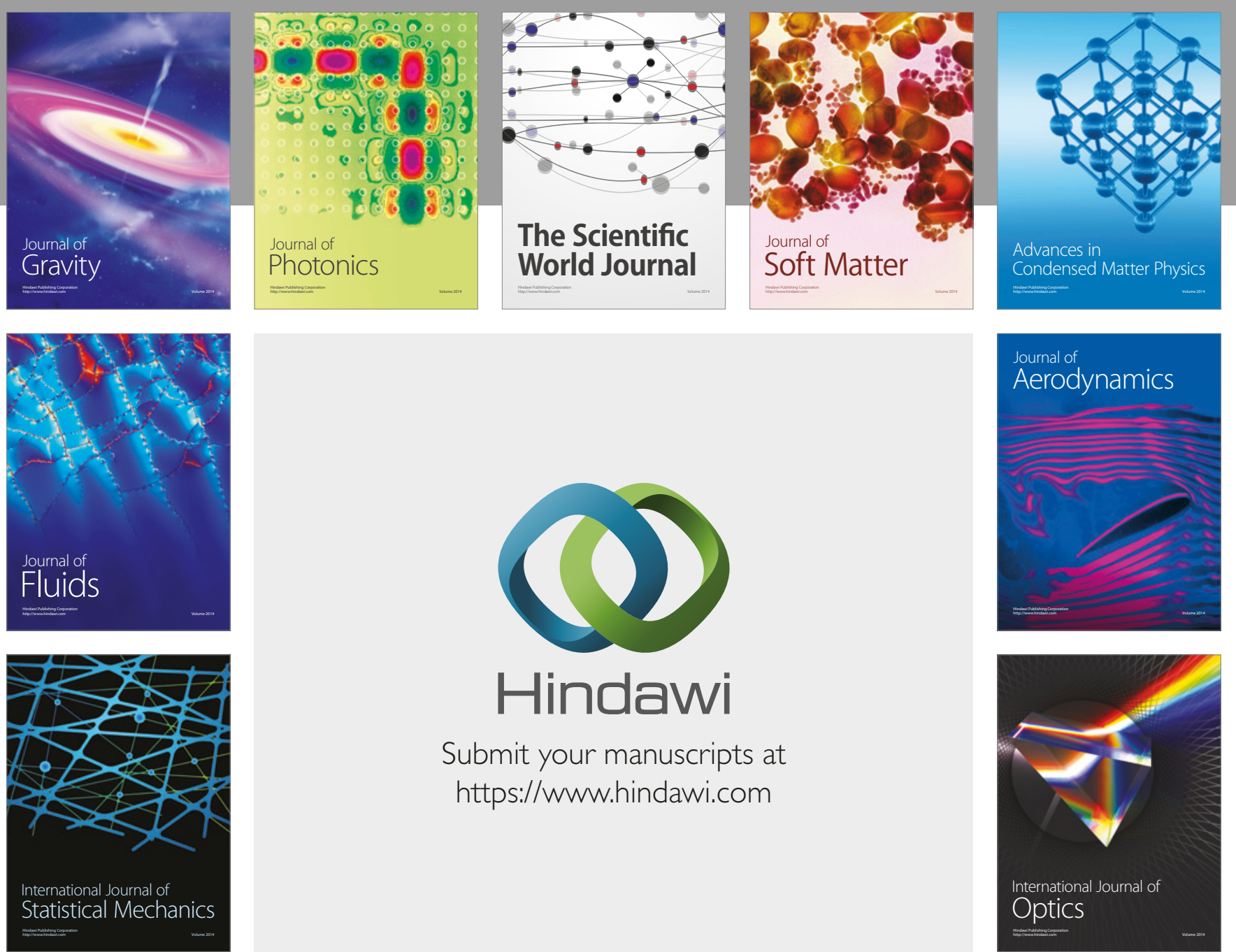

Submit your manuscripts at

https://www.hindawi.com
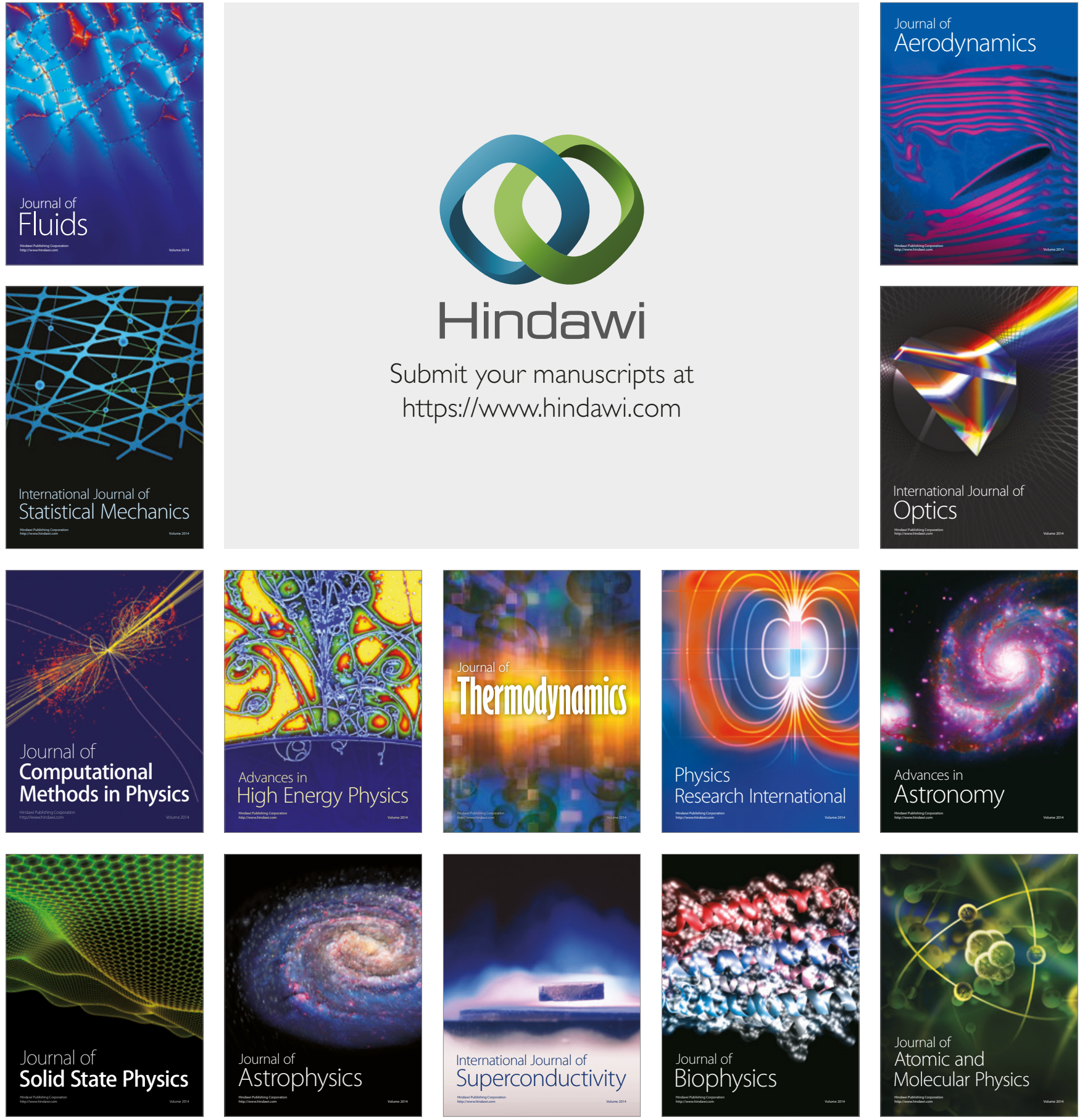